\title{
Implied Velocity and Acceleration Induce Transformations of Visual Memory
}

\author{
Ronald A. Finke \\ State University of New York at Stony Brook \\ Gary C.-W. Shyi \\ State University of New York at Stony Brook
}

Jennifer J. Freyd

Cornell University

\begin{abstract}
In this study, the phenomenon of representational momentum (Freyd \& Finke, 1984) is investigated in cases where visual memories are distorted by implied motions of the elements of a pattern. Our theory predicts that these memory distortions should be sensitive not only to the direction of the implied motions but also to changes in the implied velocity. Subjects observed a sequence of dotpattern displays that implied that the dots were moving at either a constant velocity or constant acceleration, but in separate directions. Discrimination functions for recognizing the final pattern in the sequence revealed that subjects' memories had shifted forward, corresponding to small continuations of the implied motions. The induced memory shifts increased in size as the implied velocity and acceleration of the dots increased, but were eliminated when the display sequence implied a deceleration of the dots to a final velocity of zero. These findings suggest that mentally extrapolated motion may have some of the same inertial properties as actual physical motion.
\end{abstract}

A remarkable aspect of human cognition is that cognitive processes often resemble certain physical processes. For example, studies on mental rotation by Shepard and his colleagues demonstrated that the manner in which people imagine the rotation of rigid objects resembles the way objects actually rotate (e.g., Cooper \& Shepard, 1973; Shepard \& Cooper, 1982; Shepard \& Metzler, 1971). Other examples of correspondences between cognitive and physical processes have come from studies on the imagined folding of nonrigid, two-dimensional patterns into three-dimensional forms (Shepard \& Feng, 1972), and the imagined rotation of a configuration of objects in three-dimensional space (Pinker \& Finke, 1980). According to Shepard, these correspondences are important because they suggest that the human mind has "internalized" many of the laws governing physical motion (Shepard, 1981, 1984).

Recently, Freyd and Finke (1984) reported a phenomenon that suggests there is a cognitive process analogous to the physical momentum of a moving object. They found that short-term visual memory for the final position of a geometric pattern was shifted by a preceding series of displays that implied that the pattern was rotating. Subjects were presented a stationary, out-

This research was funded by National Institutes of Health Biomedical Sciences Research Support Grant 431-H219-M and by National Institute of Mental Health Grant 1R0IMH3980901 awarded to Ronald A. Finke. Preparation of this article was partially funded by National Science Foundation Presidential Young Investigator Award BNS 8451356 awarded to Jennifer J. Freyd.

We thank Roger Shepard, Marvin Levine, Michael McCloskcy, Joan Gay Snodgrass, Mary Peterson, Mike Kelly, and Geoff Loftus for helpful comments on this article and the project and Gabriella Goldberg for her assistance in conducting the experiments.

Correspondence concerning this article should be addressed to Ronald A. Finke, Department of Psychology, State University of New York at Stony Brook, Stony Brook, New York 11794. lined rectangle at three successive orientations $17^{\circ}$ apart for durations and interstimulus intervals (ISIs) of $250 \mathrm{~ms}$, implying a smooth rotation of the rectangle in the picture plane. Following a retention period of $250 \mathrm{~ms}$, a fourth rectangle was presented, which was either identical in orientation to the third or differed by rotation of $6^{\circ}$ in the same or opposite direction as the implied rotation. This pattern was left on until the subjects indicated whether or not the last two patterns were in exactly the same position. They made almost seven times as many errors rejecting the forwardly rotated distractors and took an average of $216 \mathrm{~ms}$ longer to do so, suggesting that their visual memories had been shifted forward by the implied rotations. Because these induced forward shifts resembled the tendency for a moving object to resist attempts to halt its motion, Freyd and Finke referred to the phenomenon as representational momentum.

In a later series of experiments, Finke and Freyd (1985) found that the phenomenon could be extended to cases where the inducing displays implied a changing configuration of the elements of a pattern, depicting nonrigid transformations of its shape. For their inducing displays, they presented dot patterns in which the dots were displaced in separate directions at an average rate, in terms of degrees of visual angle, of $0.6 \%$. Their findings were similar to those of Freyd and Finke (1984): Subjects made many more errors rejecting test patterns in which the dots were displaced by a small amount $\left(0.2^{\circ}\right)$ in the same directions as in the inducing displays than in those in which the dots were displaced in the opposite, backward directions. In addition, reaction times (RTs) were $206 \mathrm{~ms}$ longer for correctly rejecting the forwardly transformed test patterns than for correctly rejecting the backwardly transformed test patterns. This is also similar to what Freyd and Finke (1984) found for implied rotations of rigid forms.

Evidence that representational momentum for implied rotation may obey some of the same laws as physical momentum was reported by Freyd and Finke (1985). They used an improved 
technique for assessing the strength of the effect, measuring shifts in the average distribution of "same" responses for a range of test pattern displacements as opposed to simply measuring differences in error rate for a single pair of forward and backward distractors. By varying the implied velocity of the inducing displays from $15 \%$ s to $49^{\circ} / \mathrm{s}$ (which was accomplished by varying the inducing ISIs from 100 to $900 \mathrm{~ms}$ while keeping the stimulus durations constant at $250 \mathrm{~ms}$ ), Freyd and Finke found that the memory shifts increased in proportion to the implied velocity at a rate of $0.019^{\circ}$ per degree $/ \mathrm{s}$ increment. This increasing shift resembled the way an object's stopping distance increases as its velocity increases because of its increasing physical momentum.

In the present study we first wanted to establish, using variations of the improved methods developed by Freyd and Finke (1985), whether the momentum effect for implied changes in a configuration of pattern elements, like that for implied rotations of rigid forms, exhibits an increasing forward shift with increases in implied velocity. If the stopping distance in representational momentum does correspond to that for physically moving objects, then implied velocity should matter even when the pattern elements are depicted to move in different directions. In particular, we should find that the memory shifts increase by an amount proportional to increases in implied velocity for each of the elements.

Our second goal was to see what would happen when the inducing displays depicted an acceleration or deceleration of the pattern elements. Again, if representational momentum is truly analogous to physical momentum, the amount of shift in visual memory should be determined by the final velocity implied by the inducing displays, not just by the average implied velocity. This is because the momentum that an accelerating or decelerating object acquires depends on its final velocity, not on its average velocity when an imparting force is applied.

We begin by presenting a theory to explain why representational momentum occurs and then consider the predictions that the theory makes for how implied motions should affect accuracy and RTs in these tasks.

\section{A Theory of Representational Momentum}

According to our theory, the induced shifts in visual memory occur because there is a natural tendency to mentally extrapolate implied motions into the future. This tendency arises for several reasons. First, it is useful in anticipating the future positions of objects that are moving in a consistent manner (e.g., Jagacinski, Johnson, \& Miller, 1983; Rosenbaum, 1975). Second, it contributes to the regulation and control of bodily movements by enabling one to anticipate how those movements should continue once they are initiated (e.g., Finke, 1979; Greenwald, 1970). Additionally, the tendency to mentally carry out implied motions is useful whenever correct recognition depends on one's ability to imagine objects moving to expected or familiar positions (e.g., Finke \& Pinker, 1983; Shepard \& Cooper, 1982).

We next assume that there is a kind of inertial property associated with the extrapolation process, which we refer to specifically as representational momentum. As a consequence of this property, mental extrapolations, like moving physical objects, cannot be instantly halted. Instead, they continue for some time after one begins to stop them. Moreover, just as the momentum of a moving object increases as its velocity increases, making the object more difficult to stop, we assume that mental extrapolations are likewise more difficult to stop as the implied velocity of the inducing displays increases. In addition, if the inducing displays imply a change in velocity, it is the final implied velocity that should matter in determining the amount of representational momentum, just as an object's physical momentum is determined by the final velocity it achieves following acceleration.

We also propose that there are important differences between physical and representational momentum. For example, in addition to the implied velocity, how the implied motions are specified can influence the amount of representational momentum. If the inducing displays are presented at too slow a rate or do not clearly depict the motions, the tendency to extrapolate will be weaker. Likewise, if the inducing displays depict a forward motion that cannot be easily extrapolated (see Freyd \& Finke, 1984, Experiment 2), representational momentum may not be created.

There are several other restrictions concerning our use of the momentum analogy that we must address. First, our proposed corresondence between physical and representational momentum pertains to how mental extrapolations are stopped, not to the particular pathways that the extrapolations might follow. Strictly speaking, these are representational pathways and may or may not correspond to the paths taken by actual physical objects. For example, mental extrapolations may carry forward imparting or constraining forces that are implied in the inducing sequence, and may thus reflect observational or conceptual biases about the motions of objects (McCloskey, Caramazza, \& Green, 1980; McCloskey \& Kohl, 1983). We would predict, however, that whichever pathway is selected in extrapolating the implied motions, representational momentum would occur along that pathway. We will elaborate upon this point later.

A related issue concerns the generality of our theory. We need to allow for the possibility that representational momentum can also occur for extrapolations that have no simple analogue to the motions of physical objects. For example, implied changes in sound, size, or color might give rise to momentum effects if these changes can be extrapolated along some representational pathway (Kelly \& Freyd, 1985). In fact, it may be possible to obtain a momentum effect for any type of implied transformation, along with an increasing forward memory shift as the rate of the implied change increases, as long as the transformation can be extrapolated into the future.

Why do we assume that mental extrapolations should exhibit an inertial tendency in the first place? It seems reasonable to do so because changes in nature usually tend to continue, especially as the rate of change increases. In the case of rigid motion, for example, objects that are moving more quickly are more likely to continue moving in the same direction in the presence of frictional or other opposing forces. It thus makes sense that the inertial properties of motion would have been internalized to some extent in the process of mental extrapolation.

There is an alternative theory that might explain our previous findings without relying on an analogy to physical momentum. Suppose that it always takes people a certain amount of time to realize that an implied motion that they have begun to extrapolate has suddenly stopped. By then, their extrapolations would have continued forward by an amount proportional to the implied 
velocity, but not because it was difficult to stop the extrapolations. This alternative account, although plausible, does not explain the momentum effect under the experimental conditions we have so far used because the final positions in the inducing sequences were the same on every trial for every subject. As a result, there was no uncertainty as to when or where the implied motions would stop, and the subjects would not have needed additional time to realize that this had occurred.

Because the memory shifts still occur even when observers know in advance when the implied motions will cease, we further assume that people can quickly stop the mental extrapolations only by applying an opposing, internal force, which we refer to as cognitive resistance. The mental act of applying cognitive resistance is analogous to the physical act of applying the brakes quickly to stop a moving car. In each case, the stopping distance will depend on the momentum and on how rapidly the resistant force is applied. We make no other proposals about the nature of cognitive resistance at this time and allow for a more elaborate characterization (and possibly a more precise term) in future refinements of the theory.

The fundamental idea underlying our theory is that even when cognitive resistance is applied with maximum effort, mental extrapolations cannot be instantly halted if there is any forward momentum still associated with them. Instead, the extrapolations will stop at some point beyond where cognitive resistance was first applied, resulting in a forward shift in the remembered final position or configuration. In contrast, if no cognitive resistance is applied, the extrapolations will continue at their normal rate to the next step in the sequence.

In all of our experiments, subjects are strongly motivated not to allow the extrapolations to continue because the task requires that they accurately recall the true final positions. If we therefore assume that cognitive resistance is applied as soon as the inducing sequence is completed and with the same maximum effort, the memory shifts will be small compared with the step size of the implied motions, and they will be proportional to the implied velocity. To return to our car analogy, if the brakes are applied forcefully as soon as the decision to stop is made, the stopping distance will be much shorter than the distance the car would have traveled if allowed to coast and will be proportional to the final velocity that the car achieved.

Exactly how large should these memory shifts be relative to the distances covered were the extrapolation to continue unimpeded? Taking the data from Freyd and Finke (1985), we found that the average memory shift for implied rotation was $12.7 \%$ of what would have been the fully extrapolated angular displacement in the next step in the inducing sequence. Assuming that the effective stopping distance would be similar for all types of mental extrapolations, we predicted that the ratio between the forward memory shifts in the present experiments and the fully extrapolated distances to the next dot positions would be around $10 \%$. Accordingly, we predicted that the actual amount of shift would increase at this same ratio as the final extrapolated distance increased.

As mentioned previously, our principal measure of the induced memory shifts was based on distributions of "same" responses given to test patterns ranging in the amount of forward and backward displacement of the dots. Following the procedure of Freyd and Finke (1985), quadratic regressions on these data provided estimates of how far the memories for the final pattern in the display sequences shifted forward. As in that study, we hoped to find that observers were most likely to regard as equivalent to the final pattern in the inducing sequence not the actual same pattern but one that was changed slightly in the direction of the implied motions.

Our second measure of the induced memory shifts was RT for responding "different" to each of the test patterns. We predicted that distributions of these RTs would also be shifted forward in proportion to the implied velocity; the maximum RT would correspond to what is remembered to be the final pattern. This follows from the general finding in the literature that discrimination time increases as the similarity between a memory representation and a presented stimulus increases (e.g., Cooper \& Podgorny, 1976).

Our third measure was RT for responding "same" to each of the test patterns. Here, we expected that the distributions would again be shifted forward by an amount proportional to the implied velocity, but with the minimum RT corresponding to the remembered final pattern. The reason is that verification time for matching the shapes of patterns generally increases as a memory representation becomes less similar to the presented stimulus (e.g., Shepard \& Cooper, 1982).

In summary, if visual memories are in fact shifted forward as described by our theory, these three measures should yield consistent results. Specifically, a test pattern that is shifted forward by approximately $10 \%$ of the fully extrapolated distance should be regarded as the remembered pattern, be accepted as such most rapidly, and take the most time to reject as being different.

\section{Experiment 1}

The major advantage of using test patterns that vary in the amount of forward and backward displacement is that they permit one to obtain better estimates of the size of the induced memory shifts. In Experiment 1, we hoped that this technique would be sensitive enough to resolve a velocity effect for implied changes in the positions of pattern elements, following the presentation of four inducing displays. Finke and Freyd (1985), using a single pair of forward and backward distractors and three inducing displays, had found only suggestive evidence for a velocity effect for these types of implied motions. We also hoped that this technique would reveal the range of individual differences in the size of the momentum effect, which we were also not able to do using our previous methods.

\section{Method}

Subjects. Sixteen undergraduate students at the State University of New York at Stony Brook served as subjects, partially satisfying a research requirement in an introductory psychology course.

Stimuli. The stimuli were generated using an IBM personal computer and displayed using an IBM color monitor. Each trial consisted of a sequence of five dot patterns containing three green dots, presented within a circular viewing field $17.6^{\circ}$ in diameter at a viewing distance of $42 \mathrm{~cm}$. The individual dots, presented as single illuminated pixels against a uniform, medium-grey background, subtended approximately 8 min of visual angle, corresponding to a distance of approximately $1 \mathrm{~mm}$ on the display screen. Additional details of the generation and calibration procedures for these stimuli can be found in Finke and Freyd (1985, Experiment 1). 
Each trial began with a red fixation dot presented at the center of the screen for $2 \mathrm{~s}$, together with a 1-s warning tone. Following a 1-s blank interval, the five dot patterns were presented in succession using an ISI of $750 \mathrm{~ms}$. The first four patterns in the sequence, the inducing displays, were presented for durations of $250 \mathrm{~ms}$ each and depicted translations of the three dots in separate directions. The presentation rate for all patterns, in terms of the stimulus-onset asynchrony (SOA), was thus one display/s. Figure 1 presents examples of these displays, which implied constant dot velocities of $1.0^{\circ} / \mathrm{s}, 0.6^{\circ} / \mathrm{s}$, and $0.2^{\circ} / \mathrm{s}$. The fourth pattern in the inducing sequence, which the subjects were to remember, was the same on every trial. The $x, y$ coordinates of the three dots in this pattern, in terms of degrees of visual angle from the center of the screen, were $(1.0,1.0),(2.0,0.0)$, and $(-2.0,-1.0)$.

To create a constant implied velocity of $1.0^{\circ} / \mathrm{s}$, the first three patterns showed the dots displaced by $3.0^{\circ}, 2.0^{\circ}$, and $1.0^{\circ}$, respectively, from their positions in the fourth pattern. Similarly, to create a constant implied velocity of $0.6 \%$, the first three patterns showed the dots displaced by $1.8^{\circ}, 1.2^{\circ}$, and $0.6^{\circ}$; and, to create a constant implied velocity of $0.2^{\circ} / \mathrm{s}$, by $0.6^{\circ}, 0.4^{\circ}$, and $0.2^{\circ}$. We decided to vary the inducing displacements and keep the inducing ISIs constant because Finke and Freyd (1985) found that subjects had difficulty attending to these types of displays whenever the inducing ISIs were lengthened sufficiently to produce the slower implied velocities. As in previous studies, implied rather than actual motion was used to avoid possible artifacts resulting from motion aftereffects. Also, the use of ISIs of $750 \mathrm{~ms}$ or longer eliminates visual apparent motion for these types of displays, as was demonstrated by Finke and Freyd (1985).

The stimuli shown in Figure 1 represent one set of inducing displays. A second set was created by showing the dots displaced by the same amounts but from different starting points and in the opposite directions, while achieving the same inducing velocities and final dot configuration. This was done to counterbalance the particular directions of implied motion.

The fifth pattern in the sequence was always a test pattern. There were seven types of test patterns; three represented forward distractors, three represented backward distractors, and the other pattern was physically identical to the last pattern in the inducing sequence. In the forward distractors, the dots were displaced $0.1^{\circ}, 0.2^{\circ}$, or $0.3^{\circ}$ in the same directions as the implied motions; in the backward distractors, they were displaced $0.1^{\circ}, 0.2^{\circ}$, or $0.3^{\circ}$ in the opposite directions. Because the two sets of inducing patterns created opposite directions of implied motion, the forward distractors for one set were the backward distractors for the other set, and vice versa. The test patterns were displayed for $3 \mathrm{~s}$ and were followed by a 4-s intertrial interval.

Procedure. The subjects were tested individually in 1-hr sessions. Subjects sat in front of the color monitor, and the nature of the task was explained. They were told that on every trial they would be shown a sequence of five dot patterns, and that they were to judge whether or not the last pattern was identical, in all respects, to the one that immediately preceded it. They were to respond to the last pattern by pressing either a "yes" or a "no" button on a response box located directly below the display.

A demonstration program then presented examples of sequences containing the "same" test pattern, a forward distractor, and a backward distractor. For these demonstrations, the implied velocity was $0.6 \%$, and the dots in the distractors were displaced by $0.2^{\circ}$. While the subjects observed these displays the experimenter pointed out the two distinct ways that the last two patterns could be different and emphasized that both types of distractors were to be regarded as different from the preceding pattern. The subjects were also told that in the actual task, the
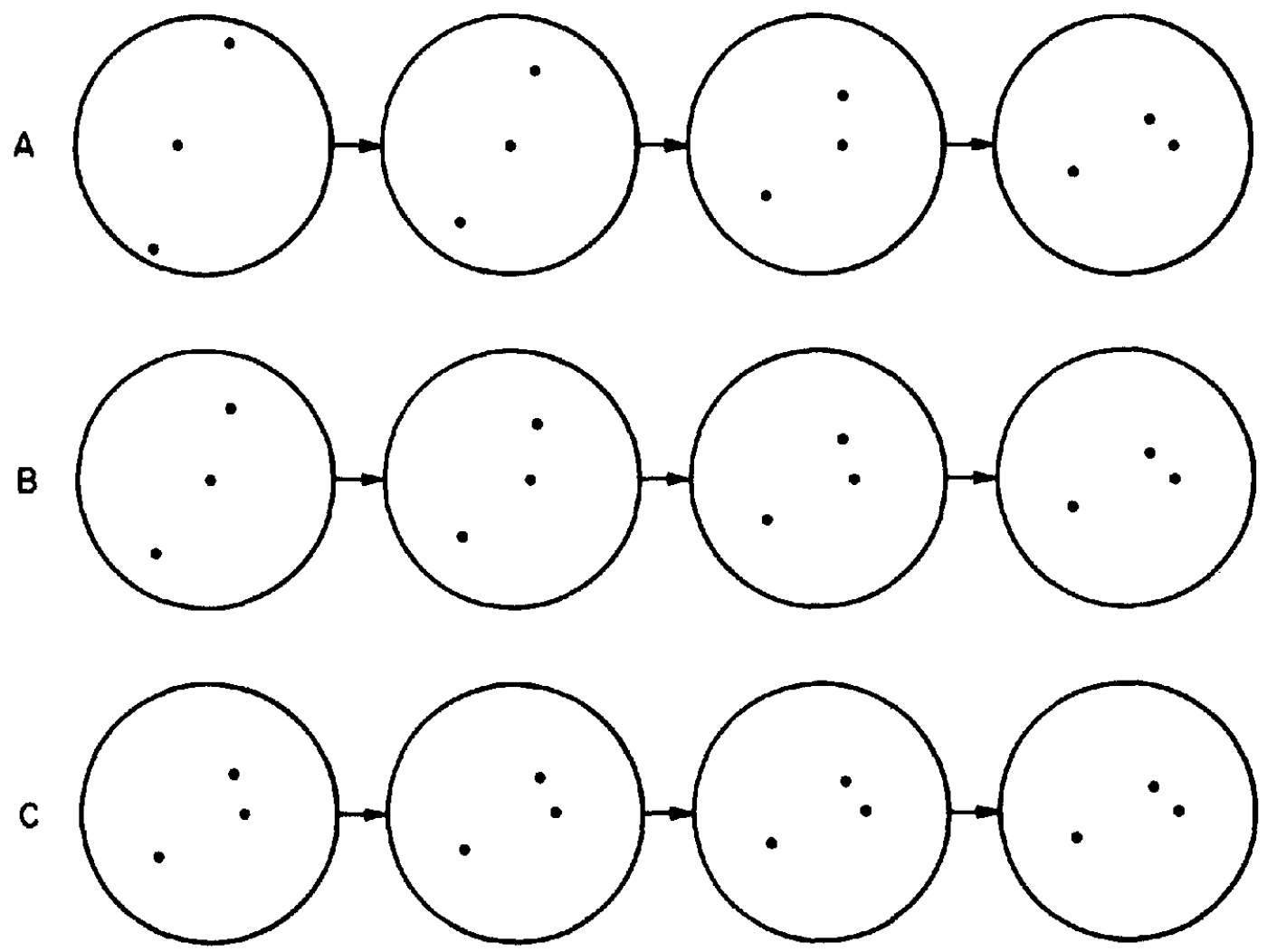

Figure 1. One set of inducing display sequences used in Experiment 1. The implied inducing velocities were (A) $1.0^{\circ} / \mathrm{s}$, (B) $0.6^{\circ} \% \mathrm{~s}$, and (C) $0.2 \%$ s. 
test patterns would sometimes be harder to judge and sometimes easier than those in the demonstration, and that the first three patterns would sometimes vary, although this would not be systematically related to the type of test pattern that would appear.

The subjects were further told that the patterns used as test patterns would be randomly selected, and that as a result there could be many trials in a row with the same correct response. In addition, because the true "same" test pattern would only appear on $14.3 \%$ of the trials, subjects were informed that although there would definitely be some truly "same" and truly "different" trials, there may be more of one kind of trial than the other. As an example, they were told the last two patterns would be identical perhaps in $80 \%$ of the trials, or, alternatively, perhaps the last two patterns would be different in $80 \%$ of the trials. This information was supplied so subjects would not just assume that half of their responses should be "same." They were also told, however, that if the last two patterns were different, there was an equal chance that the dots would be displaced in the forward and backward directions. Additional instructions stressed the importance of watching all three dots in each pattern.

The direction of implied motion was counterbalanced between subjects. There were four blocks of $\mathbf{4 2}$ trials; the first block counted as practice. Within each block, the three implied velocities and the seven types of test patterns occurred equally often. The orders of implied velocities and test patterns were randomized; the only constraint was that the same type of trial did not occur more than twice in a row. To remind the subjects when to respond, a faint audible "click" was presented in conjunction with the onset of the test patterns. At the end of each block, the subjects were given a 1 -min rest period.

Response selections and RTs were automatically recorded by the computer onto a data file. The experimenter, who was never in contact with the subjects when the trials were being conducted, monitored the equipment from a separate room. The overhead lights were dimmed prior to testing and were then turned off. At the conclusion, the subjects were debriefed regarding the purpose of the experiment and the predicted results.

\section{Results}

Analyses of variance (ANOVAs) were conducted on the number of "same" responses for the three velocity conditions and the seven types of test patterns. Shifts in the discrimination functions for these distributions were estimated using quadratic regressions. Because there were no differences between the two directions of implied motion in this or any other experiment in this study, the data were averaged across the two groups of subjects counterbalancing this factor. The RT data were not subjected to ANOVAs because many subjects did not give at least one "same" and one "different" response to each combination of implied velocity and type of test pattern. RTs greater than $3,000 \mathrm{~ms}$ or less than $100 \mathrm{~ms}$ were excluded from the reported averages and regressions; these occurred on less than $3 \%$ of all trials.

Distributions of "same" responses. Our initial analysis revealed a highly significant main effect of the type of test pattern on the average number of "same" responses, $F(6,90)=48.75$, $p<.001\left(M S_{\mathrm{e}}=3.06\right)$. As shown in Figure 2, the average distribution of "same" responses exhibited a large quadratic component, $F(1,15)=80.40, p<.001\left(M S_{\mathrm{e}}=2.11, S S=169.77\right)$, reflecting the general shape of the distribution; a smaller linear component, $F(1,15)=54.38, p<.001\left(M S_{\mathrm{e}}=1.56, S S=\right.$ 84.87), reflecting the small forward shift in the distribution; and a still smaller residual component, $F(4,60)=17.97, p<.001$ $\left(M S_{\mathrm{e}}=0.61, S S=43.85\right)$, contributing to the approximate normal form of the distribution.
The small forward shift in the average distribution was further demonstrated by contrast analyses, which revealed that there were significantly more "same" responses to each of the forward distractors than to the corresponding backward distractors ( $p<$ .001 for each analysis). A quadratic regression on these means showed that the average amount of forward shift was $0.060^{\circ}$, which was one tenth the average dot displacement in each step of the inducing sequence.

Figure 2 also presents the average distribution of "same" responses for each of the velocity conditions. As predicted, the amount of forward shift was an increasing function of implied velocity as indicated by a significant interaction between velocity and type of test pattern, $F(12,180)=7.96, p<.001\left(M S_{\mathrm{e}}=\right.$ $0.87)$. Quadratic regressions on these distributions revealed increasing forward shifts with increasing implied velocity of $0.024^{\circ}$, $0.074^{\circ}$, and $0.103^{\circ}$; these corresponded to $12.0 \%, 12.3 \%$, and $10.3 \%$, respectively, of the dot displacements in the fully extrapolated positions (which were $0.20^{\circ}, 0.60^{\circ}$, and $1.00^{\circ}$, respectively). The quadratic fits were quite good, accounting for an average of $85.3 \%$ of the variance. By comparison, linear regressions performed on these data yielded much poorer fits, accounting for an average of $21.5 \%$ of the variance.

All 16 subjects showed the momentum effect in their individual discrimination functions. For 15 subjects, the quadratic fits were reasonably good; they accounted for $52.5 \%$ to $93.3 \%$ of the individual variance and provided estimates of individual memory shifts ranging from $+0.013^{\circ}$ to $+0.175^{\circ}\left(M=+0.061^{\circ}, S D=\right.$ $0.044^{\circ}$ ). The one deviant subject gave "same" responses almost exclusively to each of the forward distractors, yielding a function that was best fitted by a linear regression (which alone accounted for $90.6 \%$ of the variance). With this single subject removed, quadratic regressions for the group data provided somewhat smaller estimated forward shifts of $0.017^{\circ}, 0.063^{\circ}$, and $0.090^{\circ}$ for the three levels of increasing implied velocity, corresponding to ratios of $8.5 \%, 10.5 \%$, and $9.0 \%$ of the fully extrapolated displacements. The subjects gave an average of $44.6 \%$ "same" responses. This ratio ranged from $30.2 \%$ to $77.0 \%$ in the individual distributions. (With the deviant subject excluded, the range was $30.2 \%$ to $61.1 \%$.)

Reaction times. The mean RTs for responding "same" in all conditions are presented in the upper part of Table 1. Quadratic regressions on these means were generally consistent with the previous analyses. They accounted for an average of $88.8 \%$ of the variance and revealed a small forward shift of $0.049^{\circ}$ for the estimated average minimum RT, which was $8.2 \%$ of the fully extrapolated displacement for the average velocity. They also revealed a velocity effect; the estimated forward shifts of $0.018^{\circ}$, $0.054^{\circ}$, and $0.068^{\circ}$ corresponded to ratios of $9.0 \%, 9.0 \%$, and $6.8 \%$ of the fully extrapolated displacements.

The lower part of Table 1 presents the average RTs for responding "different" in all conditions. As we found in our previous studies, RTs for correctly rejecting the forward distractors were, in each case, longer than those for correctly rejecting the corresponding backward distractors. Quadratic regressions accounted for an average of $88.3 \%$ of the variance and provided a larger estimate of the average memory shift than the two other measures $\left(0.096^{\circ}\right.$, which was $16 \%$ of the fully extrapolated displacement for the average velocity). The velocity effect was also larger by this measure because the estimated forward shifts for 
increasing levels of implied velocity were $0.050^{\circ}, 0.092^{\circ}$, and $0.212^{\circ}$, corresponding to ratios of $25.0 \%, 15: 3 \%$, and $21.2 \%$ of the fully extrapolated displacements.

\section{Discussion}

The results of Experiment 1 generally support our theory. The average distribution of "same" responses was an essentially normal distribution that was shifted slightly forward in the direction of the implied motions. This forward shift was also obtained for the RT functions. As expected, subjects were most likely to regard a small forward distractor as identical to the pattern they were to remember, even though the implied motions depicted changes in the pattern's shape. On the average, they were more likely to respond "same" to the $+0.1^{\circ}$ distractor than to the actual same pattern, accepted it as being the same more rapidly, and took longer to reject it as being different (see Figure 3). In addition, because the likelihood of responding "same" decreased as the forward distractor displacements increased to $+0.2^{\circ}$ and $+0.3^{\circ}$, we can rule out the possibility that the subjects continued to extrapolate the implied motions of the dots all the way forward to the next step in the inducing sequence (see also Finke \& Freyd, 1985, Experiment 4). Rather, as our theory predicts, the forward extrapolations were evidently stopped a short distance beyond the dot positions in the final inducing pattern.

Further, we found that the memory shifts, when estimated from distributions of "same" responses, increased as the implied velocity increased at approximately the same rate of $10 \%$ to $12 \%$ of the fully extrapolated displacements. This finding is consistent with that of Freyd and Finke (1985) for implied rotations of rigid forms and helps to establish the generality of the velocity effect in representational momentum. According to our theory, the memory shifts should always be proportional to the implied velocity for all types of implied motions.

An alternative explanation for our velocity effect is that subjects might have confused some of the backward distractors with the next-ta-last pattern in the inducing sequence, especially at the lowest implied velocity of $0.2^{\circ} / \mathrm{s}$. This is because the dots in that pattern were physically closer, on the average, to those in the backward distractors. As Figure 2 shows, however, subjects were no more likely to respond "same" to the $-0.2^{\circ}$ distractor when the implied velocity was $0.2^{\circ} / \mathrm{s}$ than when it was $0.6^{\circ} / \mathrm{s}$ or $1.0 \%$, even though in the former case this distractor and the next-to-last inducing pattern were identical.

The RT data provided estimates of memory shifts that were mostly consistent with those based on distributions of "same" responses because the RT distributions were each shifted forward
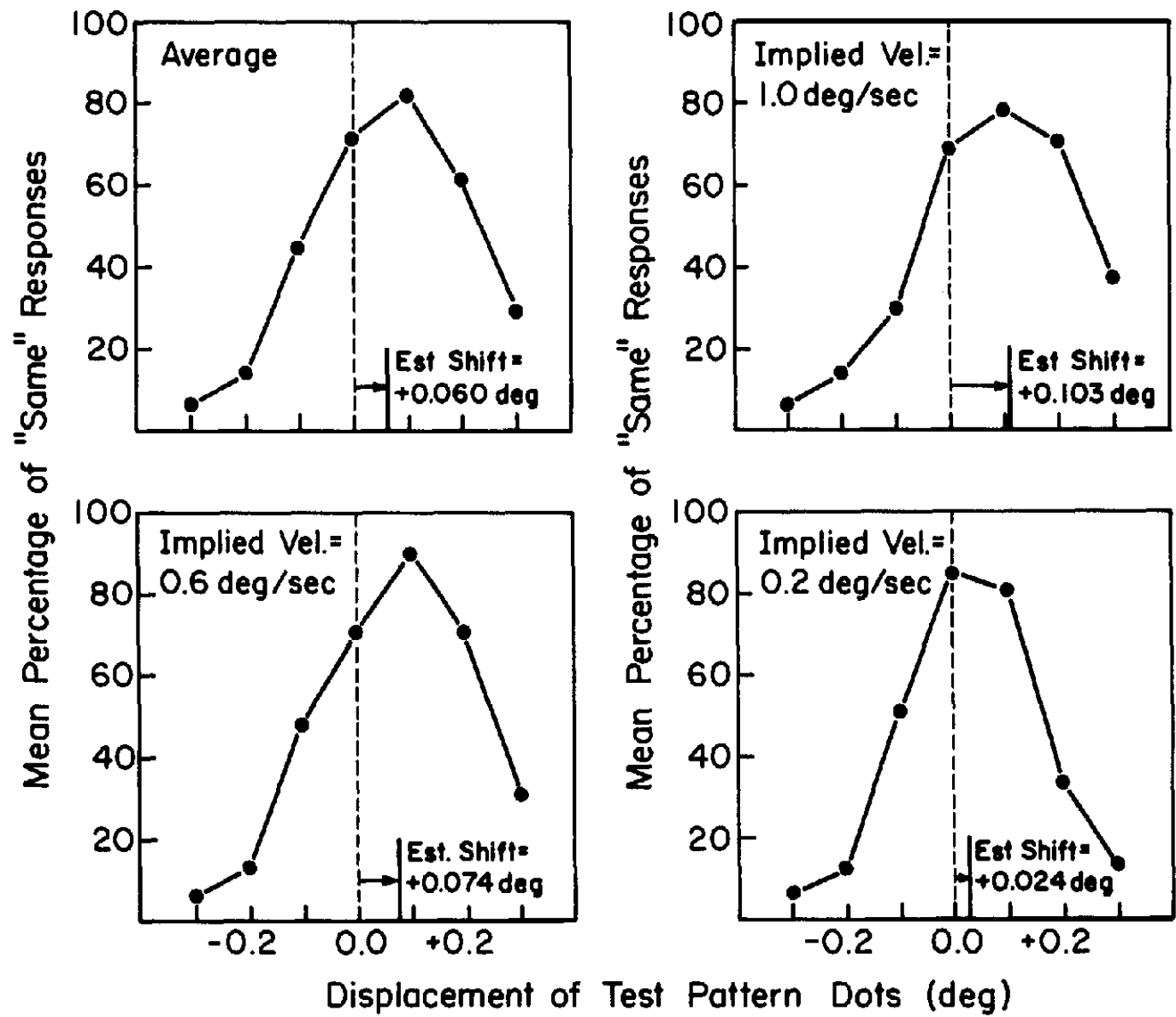

Figure 2. Mean percentage of "same" responses to each type of test pattern in Experiment 1. Dotted lines $=$ the dot positions in the actual same pattern. 
Table 1

Mean Reaction Times (in Milliseconds) in Experiment 1

\begin{tabular}{|c|c|c|c|c|c|c|c|}
\hline \multirow{2}{*}{$\begin{array}{l}\text { Implied } \\
\text { velocity }\end{array}$} & \multicolumn{7}{|c|}{ Displacement of test pattern dots } \\
\hline & $-0.3^{\circ}$ & $-0.2^{\circ}$ & $-0.1^{\circ}$ & $0.0^{\circ}$ & $+0.1^{\circ}$ & $+0.2^{\circ}$ & $+0.3^{\circ}$ \\
\hline \multicolumn{8}{|c|}{ "Same" responses } \\
\hline $1.0^{\circ} / \mathrm{s}$ & 1,593 & 998 & 971 & 1,006 & 972 & 1,019 & 1,061 \\
\hline $0.6^{\circ} / \mathrm{s}$ & 1,329 & 1,090 & 1,090 & 888 & 925 & 956 & 1,140 \\
\hline $0.2^{\circ} / \mathrm{s}$ & 1,344 & 1,085 & 1,018 & 995 & 943 & 1,232 & 1,163 \\
\hline$M$ & 1,422 & 1,058 & 1,026 & 963 & 947 & 1,069 & 1,121 \\
\hline \multicolumn{8}{|c|}{ "Different" responses } \\
\hline $1.0^{\circ} / \mathrm{s}$ & 832 & 808 & 995 & 980 & 1,082 & 1,299 & 983 \\
\hline $0.6^{\circ} / \mathrm{s}$ & 799 & 913 & 920 & 1,303 & 1,416 & 1,073 & 1,091 \\
\hline $0.2^{\circ} / \mathrm{s}$ & 829 & 889 & 1,113 & 1,201 & 1,146 & 1,042 & 990 \\
\hline$M$ & 820 & 870 & 1,009 & 1,161 & 1,215 & 1,138 & 1,021 \\
\hline
\end{tabular}

Note. Positive and negative dot displacements refer to the forward and backward distractors, respectively. The number of responses contributing to each mean may be derived from the percentages of "same" responses presented in Figure $2(N=16)$.

(see Figure 3), and because the estimated shifts increased as the implied velocity increased. However, the estimates were somewhat smaller for the "same" RTs and nearly twice as large for the "different" RTs.

The results of Experiment 1 also demonstrate that people differ considerably in the size of their memory shifts. Although each subject showed a momentum effect to some extent (ruling out the possible criticism that the mean distributions were somehow an artifact of averaging responses across subjects), estimates of forward shifts in the individual discrimination functions revealed that the magnitude of the effect varied by a factor of more than 10.

As in previous studies on the momentum effect, our findings cannot be easily explained in terms of, for example, artifactual persistence of the cathode ray tube (CRT) or sensory persistence of the icon (e.g., Coltheart, 1980; Irwin, Yantis, \& Jonides, 1983; Long, 1980; Sperling, 1960). Any residual impression of the final inducing pattern would have made it easier for subjects to reject the forward distractors.

\section{Experiment 2}

If a force acts on a moving object to change its velocity, the resultant momentum and the object's stopping distance are determined by the object's final velocity. This property of physical momentum leads to another prediction of our theory: Whenever the inducing sequence implies a consistent change in velocity, the memory shifts will be determined by the final implied velocity, not by the average implied velocity.

We therefore modified the inducing displays in Experiment 1 to represent dot patterns changing in configuration at a constant positive acceleration, a constant velocity, or a constant deceleration, while keeping the average implied velocity the same. The implied velocities in the final steps of these sequences were $1.0 \%$

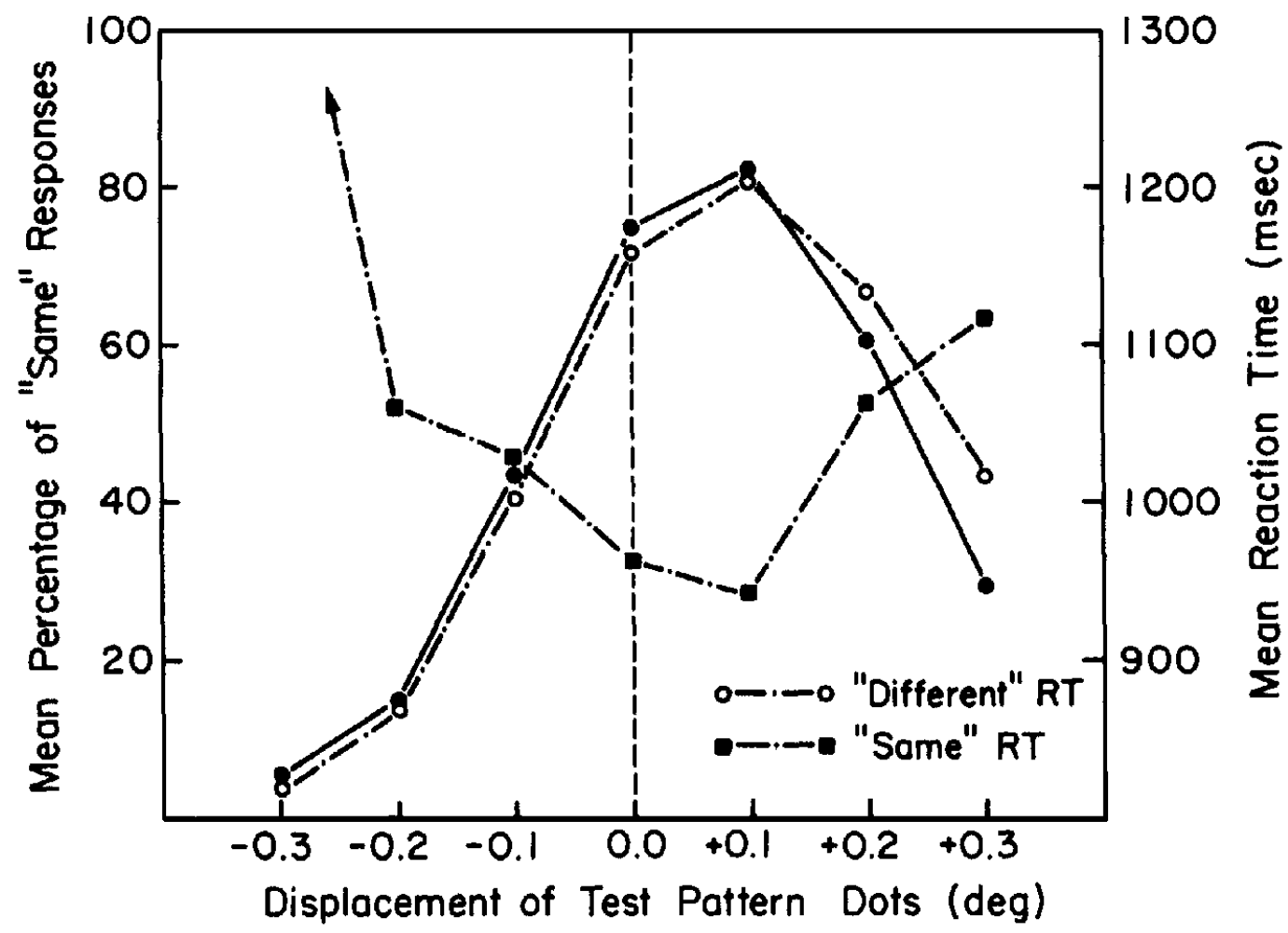

Figure 3. Comparison among distributions of the mean percentage of "same" responses (displayed by the solid line function), and the mean reaction times for "same" and "different" responses (displayed by the dashed line functions) in Experiment $1 . \mathrm{RT}=$ reaction time. 


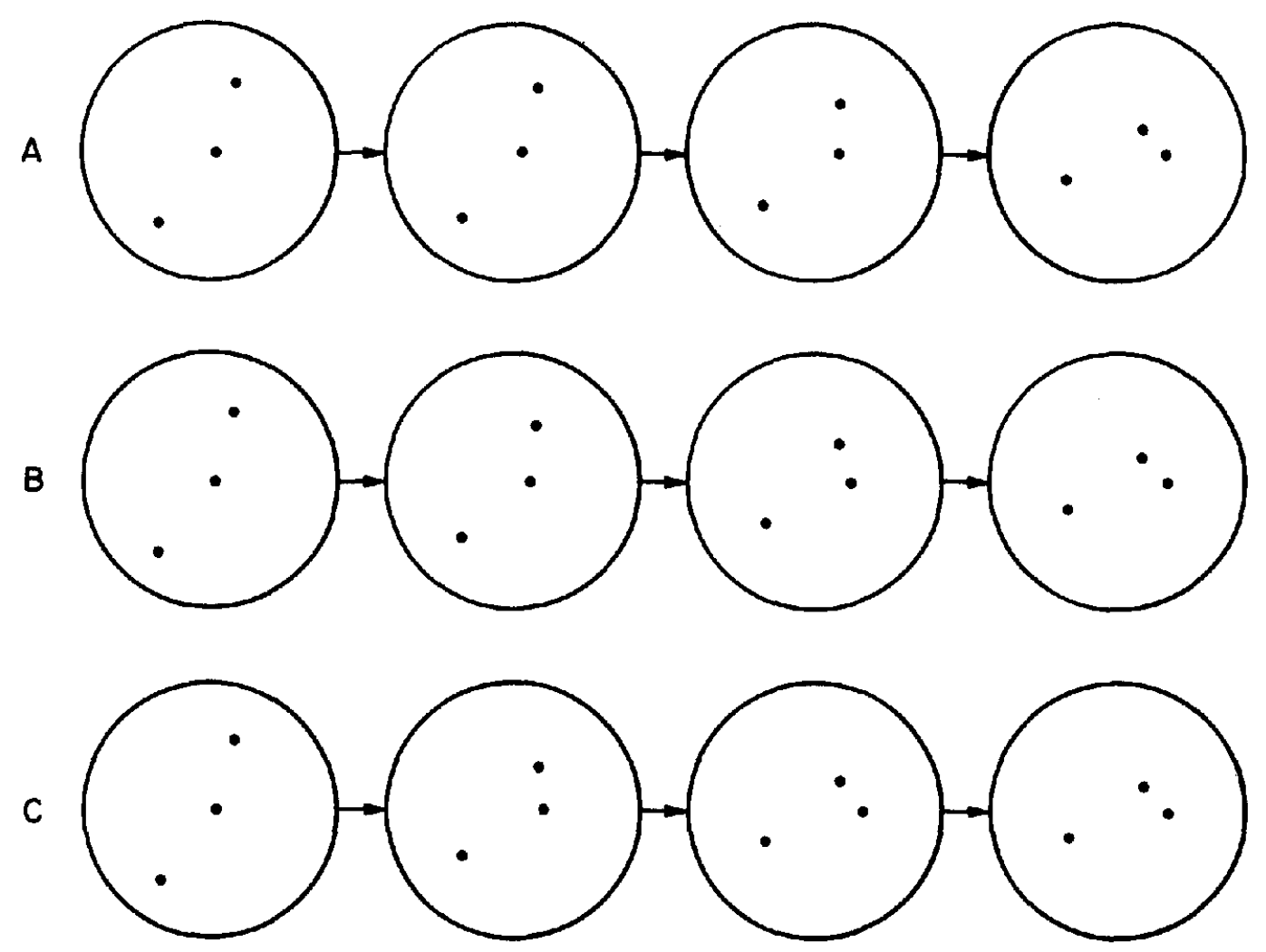

Figure 4. One set of inducing display sequences used in Experiment 2. The implied inducing accelerations were (A) $+0.4^{\circ} / \mathrm{s}^{2},(\mathrm{~B}) 0.0^{\circ} / \mathrm{s}^{2}$, and (C) $-0.4^{\circ} / \mathrm{s}^{2}$.

$\mathrm{s}, 0.6^{\circ} / \mathrm{s}$, and $0.2 \%$, respectively, matching the implied constant velocities in Experiment 1 . If representational momentum is determined by the final implied velocity, the memory shifts in the three conditions of this experiment should match those of the previous experiment. If representational momentum is determined simply by the average implied velocity across the inducing sequence, there should be no effect of the different levels of implied acceleration, and the memory shifts should be equal to the average shift in Experiment 1.

\section{Method}

Subjects. Sixteen undergraduate students at the State University of New York at Stony Brook were selected as before. None had participated in the previous experiment.

Stimuli and procedure. The inducing displays from Experiment 1 were modified as shown in Figure 4. For each set of displays, the initial and final dot configurations were identical to those for the $0.6 \% \mathrm{~s} \mathrm{constant-}$ velocity condition. For the two changing-velocity conditions, the middle two displays were adjusted to create implied constant accelerations of $+0.4^{\circ} / \mathrm{s}^{2}$ and $-0.4^{\circ} / \mathrm{s}^{2}$. This was accomplished by making the implied velocities $0.2 \% \mathrm{~s}$ and $1.0 \% \mathrm{~s}$, respectively, in the first inducing step, $0.6 \%$ $\mathrm{s}$ in the second inducing step, and $1.0^{\circ} / \mathrm{s}$ and $0.2^{\circ} / \mathrm{s}$, respectively, in the final inducing step. The average implied velocity in each condition was thus $0.6^{\circ} / \mathrm{s}$. In all other respects the stimuli and procedure were identical to those in Experiment 1.

\section{Results}

Distributions of "same" responses. As Figure 5 shows, there was a highly significant main effect of the type of test pattern on the average number of "same" responses, $F(6,90)=85.84, p<$ $.001, M S_{\mathrm{e}}=2.14$. Again, this average distribution of "same" responses displayed a predominant quadratic component, $F(1$, $15)=309.60, p<.001, M S_{\mathrm{e}}=0.81, S S=250.01$; a small linear component, $F(1,15)=80.90, p<.001, M S_{\mathrm{e}}=0.78, S S=62.75$, and a smaller residual component, $F(4,60)=20.28, p<.001$, $M S_{\mathrm{e}}=0.67, S S=54.36$. The forward distractors were regarded as "same" significantly more often than each of the corresponding backward distractors (for each comparison, $p<.001$ ). The average extent of the forward shift, estimated by a quadratic regression on the means, was $0.043^{\circ}$.

In support of our prediction that the momentum effect would be sensitive to the final implied velocity, the amount of forward shift was an increasing function of implied acceleration, $F(12$, $180)=4.17, p<.001, M S_{\mathrm{e}}=0.98$. Figure 5 presents the average distribution of "same" responses for each of the acceleration conditions; the estimated forward shifts were $0.022^{\circ}, 0.051^{\circ}$, and $0.065^{\circ}$. Assuming that the fully extrapolated distance would be determined by the final implied velocity, these shifts were $11.0 \%, 8.5 \%$, and $6.5 \%$ of the corresponding extrapolated distances, respectively. The decreasing ratios suggest an apparent constraint on the memory shifts as the final implied velocity increases. The quadratic fits were quite good, accounting for an average of $85.2 \%$ of the variance compared with $17.1 \%$ based on linear regressions.

As before, all 16 subjects displayed a forward shift in their discrimination functions. Quadratic regressions for each of these functions accounted for $49.4 \%$ to $94.5 \%$ of the individual vari- 
ance. The estimated shifts in memory ranged from $+0.017^{\circ}$ to $+0.136^{\circ}\left(M=+0.049^{\circ}, S D=0.034^{\circ}\right)$. The average proportion of "same" responses was $42.7 \%$, and these ranged from $27.8 \%$ to $56.3 \%$ across the subjects.

Reaction times. Table 2 presents, at top, the mean RTs for responding "same" in each condition. With the exception of the negative acceleration condition, initial attempts to provide quadratic fits for these means were unsuccessful; the variance accounted for averaged only $14.3 \%$. Table 2 suggests that these poor fits were mainly because of unusually fast RTs to the $-0.3^{\circ}$ distractor in the positive acceleration and constant velocity conditions. This was most likely an artifact of the small number of observations contributing to these means (one and four observations, respectively). Repeating the quadratic regressions with RTs for both the $+0.3^{\circ}$ and $-0.3^{\circ}$ distractors removed yielded much better fits: For the data averaged across all three conditions, the percentage of variance accounted for increased to $94.5 \%$, and the estimated forward shift was $0.045^{\circ}$, similar to that obtained for the distributions of "same" responses $\left(0.043^{\circ}\right)$. The estimated forward shifts for the negative acceleration, constant velocity, and positive acceleration conditions were, respectively, $0.024^{\circ}, 0.035^{\circ}$, and $0.083^{\circ}$, corresponding to $12.0 \%, 5.8 \%$, and $8.3 \%$ of the full extrapolations of the final implied velocities.
The average RTs for responding "different" to the test patterns in each condition are presented in the lower part of Table 2. The forward distractors again took longer to correctly reject than each of the corresponding backward distractors. The quadratic fits for these data were better than those for the "same" RTs, accounting for an average of $88.3 \%$ of the variance. The average estimated forward shift was $0.066^{\circ}$, and the estimated shifts for the three acceleration conditions were, respectively, $0.026^{\circ}$, $0.102^{\circ}$, and $0.140^{\circ}$, corresponding to ratios of $13.0 \%, 17.0 \%$, and $14.0 \%$ of the fully extrapolated distances. As in the previous experiment, these estimates were larger than those based on the distributions of "same" responses.

\section{Discussion}

Experiment 2 demonstrates that when there is a consistent change in the implied velocity, the extent of the memory shifts depends on the final implied velocity in the inducing sequence, not simply on the average inducing velocity. There appears, however, to be a small constraint on the rate at which the memory shifts increase. A proposed explanation for this constraint, which was not predicted by our theory, is deferred until later.

The combined results of Experiments 1 and 2 rule out the possible alternative that the momentum effect could be explained

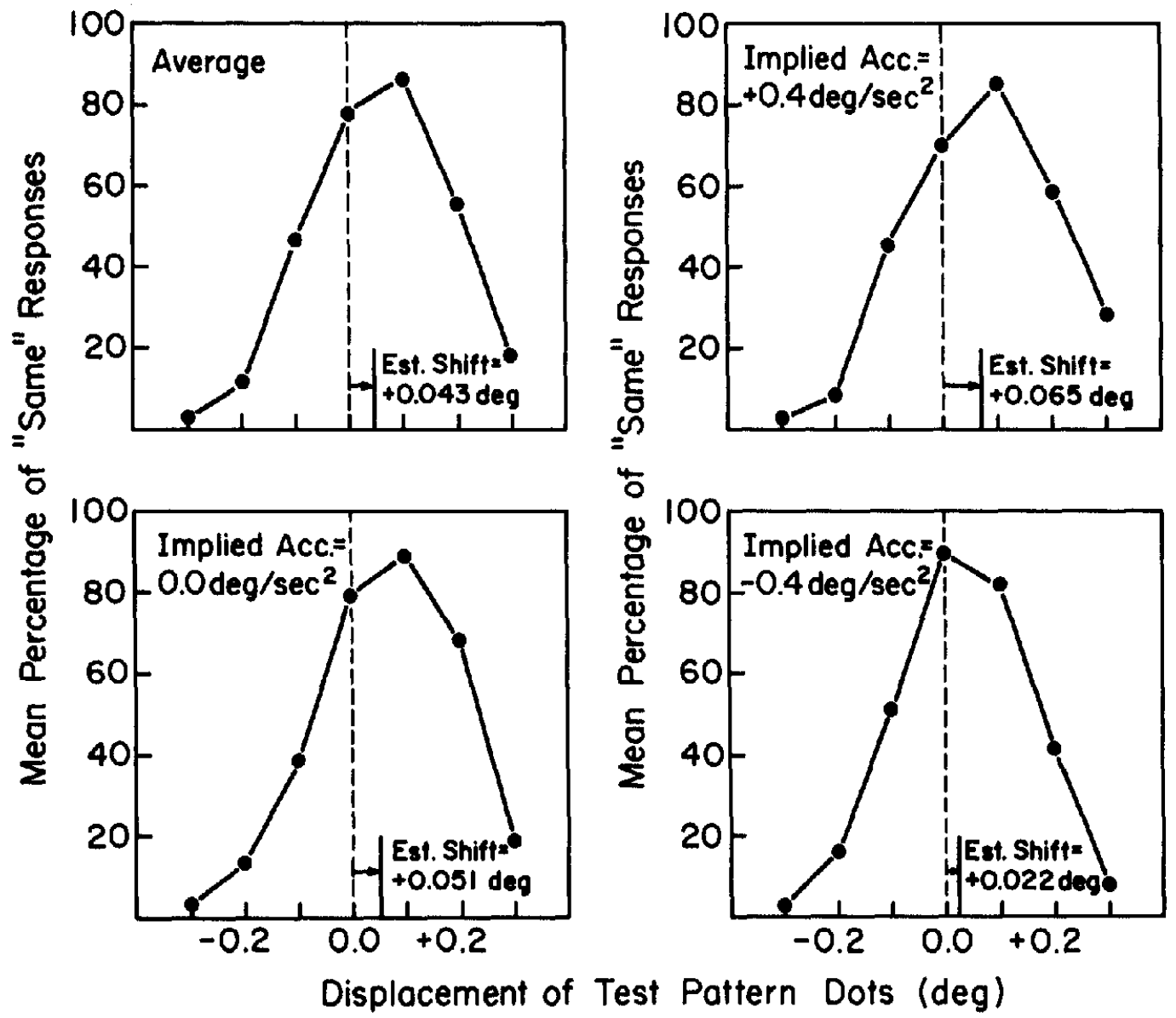

Figure 5. Mean percentage of "same" responses to each type of test pattern in Experiment 2. Dotted lines = the dot positions in the actual same pattern. For each condition, the average implied velocity was $0.6 \%$. 
Table 2

Mean Reaction Times (in Milliseconds) in Experiment 2

\begin{tabular}{|c|c|c|c|c|c|c|c|}
\hline \multirow{2}{*}{$\begin{array}{l}\text { Implied } \\
\text { acceler- } \\
\text { ation }\end{array}$} & \multicolumn{7}{|c|}{ Displacement of test pattern dots } \\
\hline & $-0.3^{\circ}$ & $-0.2^{\circ}$ & $-0.1^{\circ}$ & $0.0^{\circ}$ & $+0.1^{\circ}$ & $+0.2^{\circ}$ & $+0.3^{\circ}$ \\
\hline \multicolumn{8}{|c|}{ "Same" responses } \\
\hline $\begin{array}{r}+0.4^{\circ} / \mathrm{s} \\
0.0^{\circ} / \mathrm{s} \\
-0.4^{\circ} / \mathrm{s} \\
M\end{array}$ & $\begin{array}{r}512 \\
900 \\
1,202 \\
871\end{array}$ & $\begin{array}{r}1,205 \\
919 \\
1,131 \\
1,085\end{array}$ & $\begin{array}{l}876 \\
976 \\
948 \\
933\end{array}$ & $\begin{array}{l}863 \\
832 \\
702 \\
799\end{array}$ & $\begin{array}{l}746 \\
734 \\
805 \\
762\end{array}$ & $\begin{array}{r}836 \\
949 \\
1,007 \\
931\end{array}$ & $\begin{array}{r}805 \\
941 \\
1,064 \\
937\end{array}$ \\
\hline \multicolumn{8}{|c|}{ "Different" responses } \\
\hline $\begin{array}{c}+0.4^{\circ} / \mathrm{s} \\
0.0^{\circ} / \mathrm{s} \\
-0.4^{\circ} / \mathrm{s} \\
M\end{array}$ & $\begin{array}{l}748 \\
718 \\
700 \\
722\end{array}$ & $\begin{array}{l}742 \\
718 \\
723 \\
728\end{array}$ & $\begin{array}{l}825 \\
910 \\
896 \\
877\end{array}$ & $\begin{array}{r}987 \\
929 \\
1,167 \\
1,028\end{array}$ & $\begin{array}{r}1,014 \\
923 \\
1,073 \\
1,003\end{array}$ & $\begin{array}{l}906 \\
940 \\
819 \\
888\end{array}$ & $\begin{array}{l}922 \\
863 \\
765 \\
850\end{array}$ \\
\hline
\end{tabular}

Note. Positive and negative dot displacements refer to the forward and backward distractors, respectively. For each condition, the average implied velocity was $0.6^{\circ} / \mathrm{s}$. The number of responses contributing to each mean may be derived from the percentages of "same" responses presented in Figure $5(N=16)$.

by a tendency to increase the contrast between memories for the last two patterns in the inducing sequence. This alternative needs to be considered because, like our theory, it would have predicted small forward shifts in memory for the final inducing pattern. However, it would also have predicted that the forward shifts would increase as the last two patterns became more similar because a greater shift would then be required to make the memories distinct. This is clearly refuted by the velocity and acceleration effects obtained in these experiments because the forward shifts decreased, not increased, with increasing similarity between the last two inducing patterns (see Figures 1, 2, 4, and 5).

Another account of the velocity and acceleration effects in our first two experiments is that they might have resulted from shifts in eye position after observing the inducing displays. Although explicitly instructed not to do so, subjects might have picked out, and focused on, just one of the dots in these displays, and then tracked the dot as it was being displaced. If their eyes continued to drift slightly in the same direction, this might have caused them to misjudge the position of the dot when it was forwardly displaced in the test patterns because there were no external cues for judging the absolute positions of the dots. Accordingly, if larger implied velocities create larger shifts in eye position, our velocity and acceleration effects could have been due to this artifact.

In arguing against this account, it would have been easier for subjects to judge the test patterns according to the relative positions of the dots, and not their absolute positions because the three dots were always displaced in different directions. In addition, we conducted a simple control experiment, repeating Experiment 2 while providing a stationary fixation point together with a stationary square frame surrounding the inducing and test patterns throughout the entire presentation sequence. The presence of these stationary reference cues should have greatly reduced any possibility that subjects would misjudge the positions of the dots as a result of shifts in their eye position, and should have facilitated the detection of small displacements of the dots in the test patterns. The results of this control experiment, however, were virtually identical to those of the present experiment (the corresponding memory shifts, based on distributions of "same" responses, were $.007^{\circ}, .051^{\circ}$, and $.062^{\circ}$ ), in further support of our claim that transformations of visual memories underlie these effects.

\section{Experiment 3}

There is another explanation for why the size of the memory shifts in Experiment 2 was determined by the final and not the average implied velocity in the inducing sequence. Suppose that subjects simply did not take into account information about changing velocity provided by the first two displays. Their memory shifts would then have been determined by the implied velocity in the final inducing step, but without assuming that they were sensitive to the implied accelerations.

In fact, a closer inspection of the inducing conditions of Experiment 2 favors this alternative account. If subjects were really sensitive to the implied accelerations of the dots, their memory shifts should have been determined not by the implied velocity of the final inducing step, but rather by the final instantaneous velocity implied at the end of the inducing sequence. In the previous experiment, the final instantaneous implied velocities for the three acceleration conditions can be calculated from the formula $v($ final $)=v($ average $)+($ at $) / 2$. These were, respectively, $0.0^{\circ} / \mathrm{s}, 0.6^{\circ} / \mathrm{s}$, and $1.2 \%$. We therefore should have found no forward shift at all in our negative acceleration condition, contrary to the obtained result.

In Experiment 3, we attempted to distinguish between these two accounts by modifying the inducing display sequence to increase the salience of the implied accelerations. This was done in three ways. First, we doubled the implied accelerations and increased the average implied velocity to $1.0^{\circ} / \mathrm{s}$. Next, we increased the number of inducing displays from four to six to better depict the implied changes in velocity. Finally, we reduced the inducing ISI to $250 \mathrm{~ms}$ to shorten the integration time between the inducing displays. Although this reduction in ISI did create visual apparent motion, Finke and Freyd (1985) showed, using ISIs ranging from 250 to $500 \mathrm{~ms}$, that apparent motion does not contribute to the memory shifts.

These modifications produced implied velocities in the final inducing steps of $0.2 \% \mathrm{~s}, 1.0^{\circ} / \mathrm{s}$, and $1.8^{\circ} / \mathrm{s}$, and final instantaneous implied velocities of $0.0 \% / \mathrm{s}, 1.0^{\circ} / \mathrm{s}$, and $2.0^{\circ} / \mathrm{s}$. If the momentum effect is sensitive to the final instantaneous implied velocity, then it should disappear completely in the negative acceleration condition of this experiment where the implied changes in velocity would now be much more apparent. For the other two conditions, we expected the memory shifts to be $0.10^{\circ}$ and $0.20^{\circ}$, assuming a ratio of $10 \%$ of the full extrapolation of the instantaneous implied velocities.

\section{Method}

Subjects. Sixteen undergraduate students at the State University of New York at Stony Brook were selected as in Experiments 1 and 2.

Stimuli and procedure. The procedure of Experiment 2 was again repeated, except there were now six inducing displays, and the inducing ISIs were reduced to $250 \mathrm{~ms}$. The retention ISI remained at $750 \mathrm{~ms}$. The dot positions in the first five inducing displays were adjusted to create 
implied accelerations of $+0.8^{\circ} / \mathrm{s}^{2}, 0.0^{\circ} / \mathrm{s}^{2}$, and $-0.8^{\circ} / \mathrm{s}^{2}$; the average implied velocity in each case was $1.0^{\circ} / \mathrm{s}$. There were again two sets of inducing displays implying motions of the dots in opposite directions from different starting points. Each dot was displaced $2.5^{\circ}$ going from the initial to the final configurations.

\section{Results}

Distributions of "same" responses. Figure 6 displays the distributions of "same" responses for each condition, together with the average distribution. Whereas the distributions for the constant velocity and positive acceleration conditions were both shifted forward, as before, that for the negative acceleration condition was symmetrically distributed about the actual "same" position. Quadratic regressions, which accounted for an average of $90.4 \%$ of the variance, verified the absence of any forward shift in the negative acceleration condition (the estimated shift, as shown in Figure 6, was $-0.002^{\circ}$ ), and revealed forward shifts in the other two conditions similar to those found previously $\left(+0.061^{\circ}\right.$ and $+0.070^{\circ}$, respectively). ANOVAs yielded essentially the same pattern of results as before; there was a main effect of test pattern type, $F(6,90)=76.95, p<.001\left(M S_{\mathrm{e}}=2.21\right)$, a significant interaction between implied acceleration and type of test pattern, $F(12,180)=13.97, p<.001\left(M S_{\mathbf{e}}=.86\right)$, and significant differences in the number of "same" responses between each pair of forward and backward distractors (for each comparison, $p<.005$ ). The momentum effect was again constrained; the memory shifts in the constant velocity and positive acceleration conditions were $6.1 \%$ and $3.5 \%$ of the fully extrapolated displacements for the final instantaneous velocities.

All subjects showed the momentum effect in their individual discrimination functions. Quadratic regressions accounted for $64.5 \%$ to $96.8 \%$ of the individual variance, and the estimated memory shifts ranged from $+0.006^{\circ}$ to $+0.109^{\circ}\left(M=+0.040^{\circ}\right.$, $S D=0.031^{\circ}$ ). The average proportion of "same" responses was $46.0 \%$, and these ranged from $15.1 \%$ to $59.5 \%$ across the subjects.

Reaction times. Table 3 presents the mean RTs for responding "same" and "different" in all conditions. The absence of a momentum effect for the negative acceleration condition, and its constraint in the positive acceleration condition, are revealed by both sets of results. For the "same" RTs, quadratic regressions accounted for an average of $82.7 \%$ of the variance and provided estimated shifts, across levels of increasing implied acceleration, of $-0.031^{\circ},+0.105^{\circ}$, and $+0.061^{\circ}$, corresponding to displacement ratios of $10.5 \%$ and $3.1 \%$ in the last two conditions. For the "different" RTs, quadratic regressions accounted for an av-

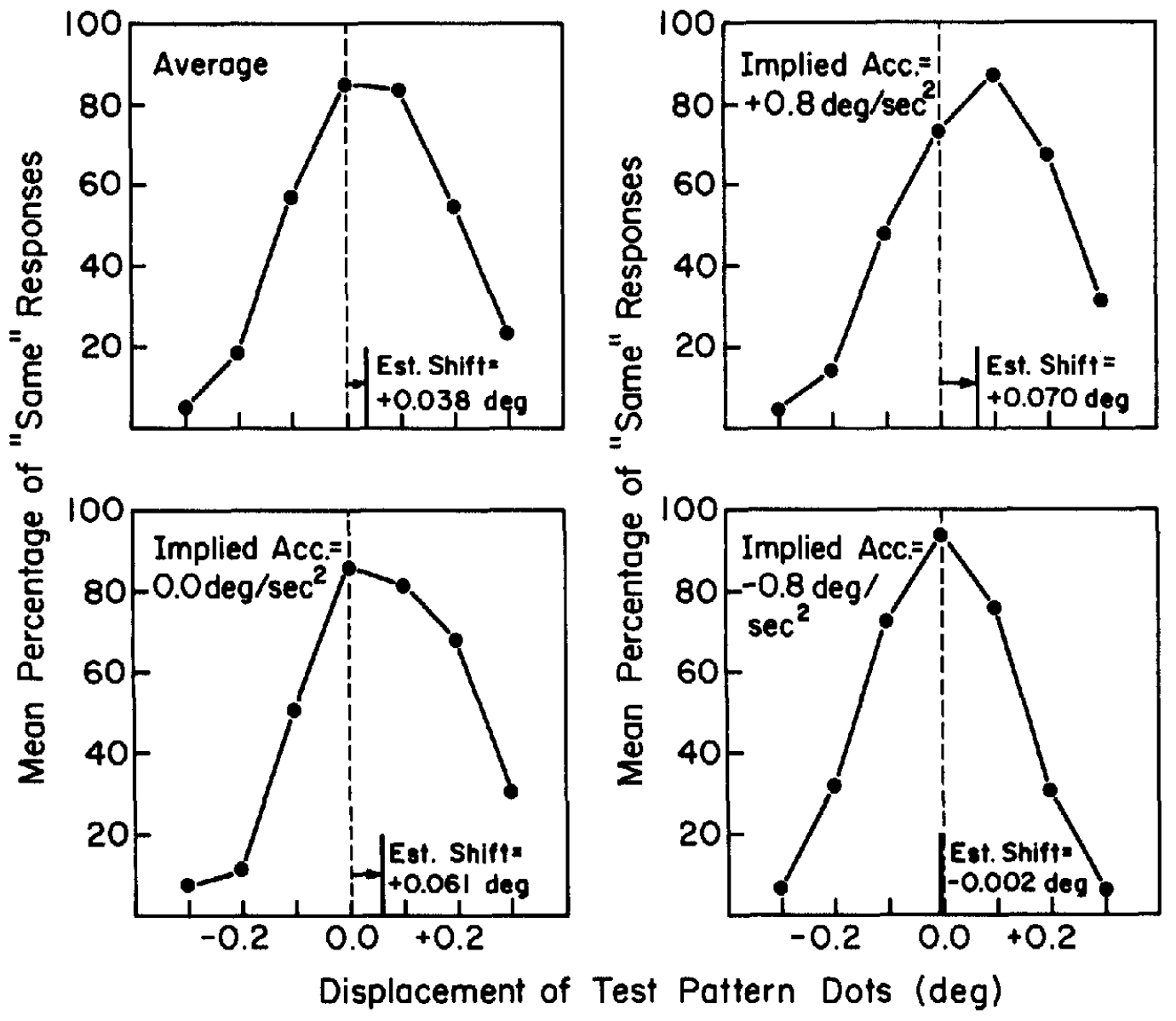

Figure 6. Mean percentage of "same" responses to each type of test pattern in Experiment 3. Dotted lines = the dot positions in the actual same pattern. For each condition, the average implied velocity was $1.0^{\circ} / \mathrm{s}(N=$ 16). 
Table 3

Mean Reaction Times (in Milliseconds) in Experiment 3

\begin{tabular}{crrrrrrr}
\hline \multirow{8}{*}{$\begin{array}{c}\text { Implied } \\
\text { acceler- } \\
\text { ation }\end{array}$} & $-0.3^{\circ}$ & $-0.2^{\circ}$ & $-0.1^{\circ}$ & $0.0^{\circ}$ & $+0.1^{\circ}$ & $+0.2^{\circ}$ & $+0.3^{\circ}$ \\
\cline { 2 - 7 } & \multicolumn{7}{c}{ "Same" responses } \\
+0.8 & 910 & 973 & 889 & 831 & 841 & 763 & 956 \\
0.0 & 1,152 & 988 & 985 & 787 & 812 & 854 & 900 \\
-0.8 & 1,211 & 981 & 800 & 747 & 959 & 883 & 1,643 \\
$M$ & 1,091 & 981 & 891 & 788 & 871 & 833 & 1,166 \\
\hline \multicolumn{7}{c}{ "Different" responses } \\
+0.8 & 700 & 805 & 983 & 1,000 & 1,282 & 1,077 & 874 \\
0.0 & 738 & 761 & 868 & 1,152 & 1,162 & 992 & 869 \\
-0.8 & 732 & 834 & 998 & 1,045 & 1,115 & 783 & 759 \\
$M$ & 723 & 800 & 950 & 1,066 & 1,186 & 951 & 834 \\
\hline
\end{tabular}

Note. Positive and negative dot displacements refer to the forward and backward distractors, respectively. For each condition, the average implied velocity was $1.0^{\circ} / \mathrm{s}$. The number of responses contributing to each mean may be derived from the percentages of "same" responses presented in Figure $6(N=16)$.

erage of $82.1 \%$ of the variance, and the estimated shifts were $+0.005^{\circ},+0.065^{\circ}$, and $+0.070^{\circ}$, corresponding to displacement ratios of 6.5 and 3.5 in the last two conditions.

\section{Discussion}

When consistent implied changes in velocity are made more salient in the inducing displays, the memory shifts are determined by the final, instantaneous implied velocity. In our negative acceleration condition, where the displays implied a constant deceleration to zero velocity, the momentum effect was completely eliminated. The effect is therefore sensitive to implied acceleration, not just to the velocity implied in the final inducing step. As in Experiment 2, however, it appears to be constrained at the higher implied velocities.

In attempting to confirm our theory, it is important to be able to show that, at least under some inducing conditions, representational momentum does not occur. In previous work, we have found that the momentum effect also disappears when the inducing displays are permuted, so that they do not imply a consistent forward motion (Freyd \& Finke, 1984, Experiment 2). That finding, along with those of the present experiment, rule out the possibility that the memory shifts occur merely as a result of prolonged observation and testing, irrespective of the nature of the inducing displays.

\section{General Discussion}

These three experiments provide evidence that the way implied motions distort visual memories resembles at least some of the lawful properties of physical momentum. Discrimination functions for recognizing the test patterns were consistently shifted in the same direction as the implied motions, and the amount of shift depended on the final implied velocity in the inducing sequence. These characteristics reflect the manner in which a physical object acquires a momentum determined by the final velocity it achieves, and support the qualitative predictions of our theory.

It has been well established that memories often change gradually over long retention periods (e.g., Bartlett, 1932) and can be distorted when biasing information is presented prior to retrieval (e.g., Loftus, 1975; Loftus, Miller, \& Burns, 1978; but see also McCloskey \& Zaragoza, 1985). However, our findings suggest a more fundamental way in which memory distortions can occur. Even though subjects were strongly motivated to remember accurately the final pattern in the sequences, the retention period was very short, and the same pattern was to be remembered on every trial, the implied motions still induced forward shifts in their memories. We would, therefore, expect such transformations to be much larger whenever implied motions are observed under natural conditions, where people would tend to be less motivated to retain a perfectly accurate memory, where the retention periods would be much longer, and where the remembered item may be seen only once (cf. Loftus, 1979; Neisser, 1982).

Our findings, consistent with those of Freyd and Finke (1985), also suggest that the best method for measuring the presence and strength of the momentum effect is to obtain discrimination functions for the likelihood of responding "same" to test patterns that are forwardly and backwardly transformed by varying amounts. Measures based on RTs for responding "same" or "different" to the test patterns appear to be somewhat less reliable, although they did yield results which for the most part were consistent with those based on distributions of "same" responses.

The quantitative predictions of our theory were only partly confirmed. In Experiment 1, we did find that the memory shifts increased at approximately the same rate in proportion to the implied velocity, amounting to $10 \%$ to $12 \%$ of the fully extrapolated distances. In Experiments 2 and 3, however, the memory shifts for the positive acceleration conditions corresponded to decreasing ratios of $6.5 \%$ and $3.5 \%$, respectively, suggesting that the memory shifts were constrained as the final implied velocity increased from $1 \%$ s to $2 \%$ s. In contrast, such constraints were not found in the study by Freyd and Finke (1985) on implied rotations of rigid forms, in which the implied angular velocity was varied from $15^{\circ} / \mathrm{s}$ to $49^{\circ} / \mathrm{s}$. Why, then, did these constraints appear in the present study?

Recent experiments by Finke and Shyi (1985) suggest that these constraints may result from a breakdown in the mental extrapolation process as the implied velocity increases, because of the more complex nature of the implied motions for these types of inducing displays. Using inducing stimuli similar to those in Experiment 1, Finke and Shyi compared performance on the memory task with that in which subjects were explicitly instructed to extrapolate the implied motions to the next step in the inducing sequence. In the context of our theory, the latter task corresponds to the case where no cognitive resistance is applied. They found that the extrapolation judgments were generally accurate up to an implied velocity of $1 \%$, but then fell behind the correct extrapolated positions as the implied velocity was further increased. Although we have not done the analogous study for implied rotations of rigid forms, it seems less likely that these same extrapolation errors would arise in that case because the implied motions are much simpler. According to 
our theory, the momentum effect should depend not only on the implied velocity, but also on how accurately the implied motions can be extrapolated. The theory does not, however, specify the precise conditions under which the extrapolations can be accurately performed.

This last point brings us to consider the relation between our work on representational momentum and that of McCloskey and his colleagues on naive physics (e.g., McCloskey, et al., 1980; McCloskey \& Kohl, 1983). Their studies showed that people often have mistaken notions about how objects would continue moving after constraining forces are removed, suggesting that there are some properties of physical momentum that are not faithfully internalized. For example, they found that people sometimes believe a ball moving within a spiral tube will continue along a curved trajectory after it emerges, thus violating the principle of inertia. Although these findings at first appear to conflict with our theoretical claims, we argue that they bear on a different aspect of the extrapolation process.

Take, for example, the case where an object is observed to move along a curved trajectory and then suddenly disappears from view. Will its motion be extrapolated along a curved path or along a straight, tangential path? Newtonian laws of physics require that the latter path will be followed in the absence of constraining forces. But if the observer assumes that the forces will continue to be applied, the extrapolation may continue along the curved path. Whichever path is chosen will therefore depend to some extent on the tacit assumptions of the observer, as McCloskey's work clearly suggests.

Our theory does not specify the particular paths that are followed in mental extrapolation. Rather, we attempt to predict what will happen along any representational pathway when one attempts to stop the extrapolation. If the extrapolation is along a curved trajectory, memory shifts will occur along the same curved trajectory. If the extrapolation is along a straight, tangential trajectory, memory shifts will occur along that trajectory. In either case, we would predict that the memory shifts will increase as the rate of extrapolation increases. We emphasize, again, that our momentum analogy properly applies to how the extrapolations are stopped, irrespective of the specific representational pathways along which the extrapolations occur. We believe, therefore, that our findings are not inconsistent with those of McCloskey.

In assessing the strength of the momentum effect in the last two experiments, we assumed that forward accelerations would be extrapolated at a rate determined by the final implied velocity. In view of the previous discussion, this may not have been a valid assumption; subjects could have continued the accelerating motions in their extrapolations. If so, their memory shifts should have been even larger than we predicted. However, because the shifts were constrained at the higher implied velocities, for reasons already considered, we cannot distinguish between these two possibilities in the present study.

A stronger version of the momentum analogy would lead to the prediction that the memory shifts should also depend on implied mass. This is because an object's physical momentum is proportional to both its mass and velocity. There are two problems, however, with extending our theory to include the effects of implied mass. The first is methodological; implied changes in mass, unlike implied changes in velocity, are difficult to control using visual displays. More important, we believe that representational momentum is a general phenomenon that can occur even when internal transformations do not correspond to the motions of physical objects. For example, Kelly and Freyd (1985) recently reported a momentum effect for implied changes in the pitch of auditory tones. It is more likely that the proper analogue to physical mass, if one exists, would be something related to the perceptual salience of the inducing displays.

We conclude by addressing two remaining questions. First, one might ask why people could not both extrapolate implied motions forward and, at the same time, try to remember the final display in the inducing sequence. It is certainly possible, for example, that a forward extrapolation could be stopped at one level of memory, with a resulting momentum effect, while being continued at some other level. However, our theory would predict that any attempt to do so would reduce the amount of cognitive resistance and would thus tend to increase the size of the momentum effect.

Finally, one could ask why people do not merely apply cognitive resistance sooner during the inducing sequence, the same way they apply the brakes well in advance of where they want a car to stop. The reason, we believe, is that people ordinarily are not aware of the consequences of representational momentum, unlike those of a car's momentum. Hence, we think that it might be possible for someone to learn to compensate for the momentum effect after being properly informed about it and extensively trained, in much the same way that one might learn to compensate for certain types of visual illusions.

\section{References}

Bartlett, F. C. (1932). Remembering. Cambridge, England: Cambridge University Press.

Coltheart, M. (1980). Iconic memory and visual persistence. Perception \& Psychophysics, 27, 183-228.

Cooper, L. A., \& Podgorny, P. (1976). Mental transformations and visual comparison processes: Effects of complexity and similarity. Journal of Experimental Psychology: Human Perception and Performance, 2, 503514.

Cooper, L. A., \& Shepard, R. N. (1973). Chronometric studies of the rotation of mental images. In W. G. Chase (Ed.), Visual information processing (pp. 75-176). New York: Academic Press.

Finke, R. A. (1979). The functional equivalence of mental images and errors of movement. Cognitive Psychology, 11, 235-264.

Finke, R. A., \& Freyd, J. J. (1985). Transformations of visual memory induced by implied motions of pattern elements. Journal of Experimental Psychology: Learning, Memory, and Cognition, 11, 780-794.

Finke, R. A., \& Pinker, S. (1983). Directional scanning of remembered visual patterns. Journal of Experimental Psychology: Learning, Memory, and Cognition, 9, 398-410.

Finke, R. A., \& Shyi, G. C.-W. (1985). Mental extrapolation and representational momentum for implied motions. Manuscript in preparation.

Freyd, J. J., \& Finke, R. A. (1984). Representational momentum. Journal of Experimental Psychology: Learning, Memory, and Cognition, 10, 126-132.

Freyd, J. J., \& Finke, R. A. (1985). A velocity effect for representational momentum. Bulletin of the Psychonomic Society, 23, 443-446.

Greenwald, A. G. (1970). Sensory feedback mechanisms in performance control: With special reference to the ideomotor mechanism. Psychological Review, 77, 73-99.

Irwin, D. E., Yantis, S., \& Jonides, J. (1983). Evidence against visual integration across saccadic eye movements. Perception \& Psychophysics, $34,49-57$. 
Jagacinski, R. J., Johnson, W. W., \& Miller, R. A. (1983). Quantifying the cognitive trajectories of extrapolated movements. Journal of Experimental Psychology: Human Perception and Performance, 9, 43S7.

Kelly, M. H., \& Freyd, J. J. (1985). Explorations of representational momentum. Submitted for publication.

Loftus, E. F. (1975). Leading questions and the eyewitness report. Cognitive Psychology, 7, 560-572.

Loftus, E. F. (1979). Eyewitness testimony. Cambridge, MA: Harvard University Press.

Loftus, E. F., Miller, D. G., \& Burns, H. J. (1978). Semantic integration of verbal information into a visual memory. Journal of Experimental Psychology: Human Learning and Memory, 4, 19-31.

Long, G. M. (1980). Iconic memory: A review and critique of the study of short-term visual storage. Psychological Bulletin, 88, 785-820.

McCloskey, M., Caramazza, A., \& Green, B. (1980). Curvilinear motion in the absence of external forces: Naive beliefs about the motions of objects. Science, 210, 1139-1141.

MoCloskey, M., \& Kohl, D. (1983). Naive physics: The curvilinear impetus principle and its role in interactions with moving objects. Journal of Experimental Psychology: Learning, Memory, and Cognition, 9, 146156.

McCloskey, M., \& Zaragoza, M. (1985). Misleading postevent information and memory for events: Arguments and evidence against memory impairment hypotheses. Journal of Experimental Psychology: General, $114,1-16$.
Neisser, U. (1982). Memory observed: Remembering in natural contexts. San Francisco: Freeman.

Pinker, S., \& Finke, R. A. (1980). Emergent two-dimensional properties of images rotated in depth. Joumal of Experimental Psychology: Human Perception and Performance, 6, 244-264.

Rosenbaum, D. A. (1975). Perception and extrapolation of velocity and acceleration. Journal of Experimental Psychology: Human Perception and Performance, 1, 395-403.

Shepard, R. N. (1981). Psychophysical complementarity. In M. Kubovy \& J. R. Pomerantz (Eds.), Perceptual organization (pp. 279-341). Hillsdale, NJ: Erlbaum.

Shepard, R. N. (1984). Ecological constraints on internal representation: Resonant kinematics of perceiving, imagining, thinking, and dreaming. Psychological Review, 91, 417-447.

Shepard, R. N., \& Cooper, L. A. (1982). Mental images and their transformations. Cambridge, MA: M.I.T. Press.

Shepard, R. N., \& Feng, C. (1972). A chronometric study of mental paper folding. Cognitive Psychology, 3, 228-243.

Shepard, R. N., \& Metzler, J. (1971). Mental rotation of three-dimensional objects. Science, 171, 701-703.

Sperling, G. (1960). The information available in brief visual presentations. Psychological Monographs, 74 (11, Whole No. 498).

Received August 26, 1985

Revision received December 12, 1985 